\title{
Aspectos sociofamiliares de la población inmigrante en Cataluña
}

\author{
MD. Riera Vidala , MC. Baraza Mendoza ${ }^{b}$, RM. Masvidal Aliberch ${ }^{b}$, E. de Frutos Gallego, \\ A. Estabanell Buxóc, C. Cruz Rodríguez ${ }^{d}$, B. Miguel Gile, C. Guzmán Molina ${ }^{a}$ \\ -Pediatra. CAP Raval Sud. Barcelona. España. \\ ${ }^{\circ}$ Pediatra. CAP Dr. Lluís Sayé. Barcelona. España. \\ ‘Pediatra. CAP Gòtic. Barcelona. España. \\ ${ }^{\mathrm{E} E n f e r m e r a . ~ C A P ~ G o ̀ t i c . ~ B a r c e l o n a . ~ E s p a n ̃ a . ~}$

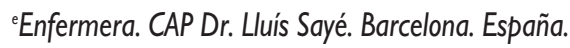

Rev Pediatr Aten Primaria. 2009; I :439-50

M. ${ }^{a}$ Dolors Riera Vidal, I19/3mrv@comb.cat

\section{Resumen}

Objetivos: conocer algunas características sociofamiliares de la población inmigrante que llega a las consultas pediátricas de Cataluña.

Material y métodos: estudio prospectivo, transversal y multicéntrico. Población: niños entre 6 meses y 15 años que hacía menos de un año que habían llegado a Cataluña procedentes de un país de baja renta (PBR). Variables: sexo, edad, país de procedencia, consanguinidad de los padres, fecha de migración y de primera visita, cuidador principal, familia completa o no, índice de hacinamiento, religión familiar y estudios de los padres.

Resultados: se estudiaron 1.226 pacientes, 659 (53,8\%) varones y 567 (46,2\%) mujeres. Procedían de 47 países, agrupados en 9 zonas de procedencia. Media de edad de la muestra: 7,5 años. Mediana del tiempo entre la llegada a Cataluña y la primera visita al centro de asistencia primaria: 1 mes. Cuidador principal: madre o padre en el 96,9\%. El 70,9\% tenían la familia nuclear completa. Consanguinidad entre los padres: 13,2\% de los casos. El mayor hacinamiento (índice de hacinamiento [IH] > 2) se da en familias indopakistaníes (49,7\%), magrebies (41,6\%) y otros países de Asia (41,8\%). Religión familiar: musulmana en el 45,9\% de los casos, católica en el 38,8\% y otras confesiones cristianas en el 10,5\%. No habían ido a escuela: $5,5 \%$ de los padres y $16,4 \%$ de las madres.

Conclusiones: las particularidades culturales y sociales de la población inmigrante hacen necesario su conocimiento para poder ofrecer la mejor asistencia posible a este colectivo.

Palabras clave: Inmigración, Diversidad cultural, Infancia, Consanguinidad, Vivienda, Educación.

El Departament de Salut de la Generalitat de Catalunya (Pla Director d'Inmigració) dio soporte institucional y económico al proyecto. Ninguno de los participantes en dicho estudio ha recibido retribución por ello, utilizándose el soporte económico para la elaboración de los datos estadísticos, la secretaría y reprografía, así como para formación. 
Abstract

Objectives: to acquire a better understanding of socio-familiar features of the immigrant population attended in the pediatric offices in Catalonia.

Methodology: prospective, transversal and multicentric study. Population: children aged 6 months to 15 years, who had arrived to Catalonia less than one year before, and who were of low income countries' origin. Variables: sex, age, country of origin, blood relationship between the parents, date of migration and first visit to the medical office, main caregiver, complete/non-complete family, home overcrowding index, religion and parents' education.

Results: one thousand two hundred and twenty-six patients were studied, 659 males (53.8\%) and 567 females (46.2\%). They had come from 47 countries, which were grouped into 9 different "origin" groups. Average age of the sample: 7.5 years. Average time between arrival to Catalonia and first visit to the doctor: 1 month. Main caregiver: mother or father in $96.9 \%$ of the cases. In $70.9 \%$ of the cases, the family was complete. Parents' blood relationship: $13.2 \%$ of the cases. The biggest home overcrowding index $(\mathrm{IH}>2)$ was founded among Indian-Pakistan (49.7\%), Maghreb (41.6\%) and other Asian (41.8\%) families. Family religion: Muslims (45.9\%), Catholic (38.8\%) and other Christian doctrines (10.5\%). Five point five percent of parents and $16.4 \%$ of mothers had never gone to school.

Conclusions: in order to provide the best medical assistance to immigrants, it is necessary to have a good understanding of their cultural and social background.

Key words: Immigration, Cultural diversity, Childhood, Consanguinity, Housing, Education.

\section{Introducción}

La importante afluencia de inmigrantes que se ha producido en nuestro país plantea nuevas exigencias al sistema sanitario, que ha de atender nuevos problemas de salud y de acceso a los servicios, tanto en aspectos concernientes solo al individuo como a la salud públi$\mathrm{Ca}^{1,2}$.

La OMS define la salud como el estado de completo bienestar físico, psíquico y social, y no solo la ausencia de enfermedad. En Pediatría es conocido que la familia y el entorno son especialmente importantes para el correcto desarrollo del niño, por lo que la llegada a nuestro país de familias inmigrantes plantea un reto en Atención Primaria (AP), debido princi- palmente a sus diferencias culturales y religiosas ${ }^{3}$, condicionantes de hábitos de vida desconocidos por nosotros, especialmente cuando provienen de países de renta baja (PRB) ${ }^{4,5}$.

Mientras que en el año 2001 la población inmigrante en España era del 3\%, en 2007 había ascendido al 8,79\% ${ }^{6}$. En Cataluña, a finales de 2005, momento en que comienza nuestro estudio, la población extranjera estimada era del $10 \%$. Por otra parte, la inmigración en Cataluña presenta una mayor diversidad y un mayor porcentaje de procedencia extracomunitaria (77\% en 2007) que en el conjunto de España, con un mayor componente, por tanto, de inmigración económica ${ }^{7}$. A los datos oficiales habría que 
añadir las cifras de los inmigrantes cuya situación no está regularizada, pero que son usuarios de los servicios sanitarios ${ }^{4,8}$.

En Cataluña, el "Protocol d'Atenció a Infants Immigrants" (PAll) ${ }^{9}$ especifica los cribados a realizar a los niños procedentes de PBR, en relación con medidas preventivas y patologías de riesgo según país de origen. En el año 2006, se realizó un estudio de efectividad de dicho protocolo, uno de cuyos objetivos fue conocer mejor ciertas características sociofamiliares de la población infantil recién inmigrada, para atenderla de forma adecuada y contextualizar la problemática detectada en la aplicación del PAll.

\section{Pacientes y métodos}

Tipo de estudio: prospectivo, transversal y multicéntrico.

Población: niños y niñas entre 6 meses y 15 años de edad, procedentes de PBR, llegados a Cataluña en los 12 meses anteriores a la primera visita realizada por alguno de los profesionales de Pediatría que colaboraron en el estudio, entre el 1 de diciembre de 2005 y el 1 de diciembre de 2006.

Participaron en el estudio un total de 69 pediatras y 64 enfermeras de AP de 44 centros de AP de toda Cataluña.

Se consideraron PBR todos, excepto: Estados Unidos de América, Canadá, Ja- pón, Nueva Zelanda, Australia y los de Europa Occidental.

Variables: sexo, edad, país de procedencia, consanguinidad de los padres (sí/no/grado), niño adoptado (sí/no), fecha de la migración y de la primera visita, religión de la familia, cuidador principal, índice de hacinamiento (definido como el cociente entre el número de personas que conviven en un domicilio y el número de habitaciones del domicilio, excluyendo cocina y baño; cuando el resultado es superior a 2 se considera que el hogar se encuentra en condiciones de hacinamiento) ${ }^{10}$, familia completa (sí/no), estudios realizados por los padres.

En el caso de los niños adoptados, no se registraron las variables correspondientes a la familia.

Para facilitar el estudio se agruparon los países de procedencia de la siguiente manera: Magreb, África subsahariana, Indopakistán, América Central, Caribe, América del Sur, otros países de Asia, antigua URSS y resto de Europa del Este.

Fueron criterios de exclusión la negativa de los padres a contestar la encuesta y la procedencia de zonas de PRB de características socioeconómicas similares a los países de renta alta.

Los datos fueron recogidos mediante una encuesta aplicada por los profesionales participantes. Previamente se re- 
dactó un libro de instrucciones para unificar criterios y se realizó una prueba piloto parar comprobar la idoneidad de la encuesta. Las respuestas fueron introducidas en una base de datos (Easytrials ${ }^{\circledast}$ ) comunicada a través de Internet.

Se realizó un estudio descriptivo con etiquetado y control de la cualidad de los datos. El análisis descriptivo de las variables cuantitativas y/o categóricas incluyó: media, mediana, moda, rango, desviación típica, percentil p25 y p75, proporciones y pruebas de normalidad. Las prevalencias se describieron de forma global y estratificada por edad, sexo y país de origen.

Para la comparación de proporciones se aplicó la prueba estadística Ji cuadrado y/o prueba exacta de Fisher, según cumplimiento de las condiciones de aplicación de cada una de ellas. Las variables cuantitativas se compararon mediante la prueba $t$ de student o $U$ de Mann-Whitney si no cumplían los supuestos de normalidad.

El paquete estadístico utilizado fue SPSS ${ }^{\circledast}$ para PC versión 13.

\section{Resultados}

Se estudiaron un total de $1.226 \mathrm{pa}-$ cientes, $659(53,8 \%)$ varones y 567 $(46,2 \%)$ mujeres. La media de edad de la muestra fue de 7,5 años (intervalo de confianza [IC] 95\%: 7,2-7,7; desviación estándar [DS]: 4,06). El 50\% de los niños/as tenían menos de 8 años y la moda fue de 11 años (8,8\%); 60 (4,89\%) eran adoptados.

La procedencia de los niños se muestra en la tabla I. Hay que destacar que la proporción de varones es máxima entre los procedentes de Asia $(58,4 \%)$, sobre todo a expensas de los nacidos en Bangladesh y Pakistán; y mínima en los procedentes de América (51,8\%), siendo la diferencia significativa según el país de origen $(p=0,0001)$.

La media del tiempo transcurrido entre la llegada a Cataluña y la primera visita al CAP fue de 1,5 meses (IC 95\%: 1,45$1,65)$ en los niños no adoptados y de 0,7 entre los adoptados $(p<0,0001)$. La mediana en el conjunto de la muestra fue de 1 mes.

La persona cuidadora principal del niño/a fue la madre o el padre en 1.111 $(96,9 \%)$ casos, en $35(3,1 \%)$ se encargaban otras personas. La distribución de los cuidadores por zona de procedencia se refleja en la tabla II. La diferencia, según el país de procedencia, es estadísticamente significativa $(p<0,0001)$.

El 70,9\% de los niños tenían la familia completa: madre, padre y hermanos no emancipados (tabla III). 
Tabla I. Distribución de los niños inmigrantes según el país de procedencia $(n=1.210)$

\begin{tabular}{|c|c|c|c|}
\hline Región & $\mathrm{n}$ & País & $\mathrm{n}$ \\
\hline \multirow[t]{2}{*}{ Magreb } & $285(23,6 \%)$ & Marruecos & $278(23,0 \%)$ \\
\hline & & Argelia & $7(0,6 \%)$ \\
\hline \multirow[t]{7}{*}{ África subsahariana } & $41(3,4 \%)$ & Senegal & $7(0,6 \%)$ \\
\hline & & Gambia & $7(0,6 \%)$ \\
\hline & & Etiopía & $6(0,5 \%)$ \\
\hline & & Guinea & $5(0,4 \%)$ \\
\hline & & Mali & $4(0,3 \%)$ \\
\hline & & Ghana & $3(0,2 \%)$ \\
\hline & & Otros & $9(0,8 \%)$ \\
\hline \multirow[t]{3}{*}{ Indopakistán } & $185(15,3 \%)$ & Pakistán & $125(10,4 \%)$ \\
\hline & & Bangladés & $45(3,7 \%)$ \\
\hline & & India & $15(1,2 \%)$ \\
\hline \multirow[t]{4}{*}{ Otros países de Asia } & $77(6,3 \%)$ & Filipinas & $35(2,9 \%)$ \\
\hline & & China & $34(2,8 \%)$ \\
\hline & & Nepal & $7(0,6 \%)$ \\
\hline & & Tadjikistán & $1(0,1 \%)$ \\
\hline \multirow[t]{3}{*}{ América Central } & $60(5,0 \%)$ & Honduras & $54(4,5 \%)$ \\
\hline & & Guatemala & $3(0,2 \%)$ \\
\hline & & Otros & $3(0,2 \%)$ \\
\hline \multirow[t]{3}{*}{ Caribe } & $45(3,7 \%)$ & R. Dominicana & $35(2,9 \%)$ \\
\hline & & Dominica & $6(0,5 \%)$ \\
\hline & & Otros & $4(0,3 \%)$ \\
\hline \multirow[t]{10}{*}{ América del Sur } & $338(27,9 \%)$ & Bolivia & $116(9,6 \%)$ \\
\hline & & Colombia & $55(4,5 \%)$ \\
\hline & & Brasil & $46(3,8 \%)$ \\
\hline & & Ecuador & $34(2,8 \%)$ \\
\hline & & Argentina & $24(2,0 \%)$ \\
\hline & & Chile & $23(1,9 \%)$ \\
\hline & & Perú & $16(1,3 \%)$ \\
\hline & & Uruguay & $12(1,0 \%)$ \\
\hline & & Venezuela & $6(0,5 \%)$ \\
\hline & & Paraguay & $6(0,5 \%)$ \\
\hline \multirow[t]{4}{*}{ Antigua URSS } & $43(3,6 \%)$ & Rusia & $18(1,6 \%)$ \\
\hline & & Ucrania & $14(1,2 \%)$ \\
\hline & & Moldavia & $5(0,4 \%)$ \\
\hline & & Otros & $6(0,4 \%)$ \\
\hline \multirow[t]{2}{*}{ Europa del Este } & $136(11,2 \%)$ & Rumanía & $124(10,2 \%)$ \\
\hline & & Bulgaria & $6(0,5 \%)$ \\
\hline
\end{tabular}


Tabla II. Características de los cuidadores del niño inmigrante en nuestro medio, según zona de procedencia $(n=1.146)$

\begin{tabular}{lccc}
\hline Procedencia & Padre y/o madre & Otras personas & $\mathbf{n}$ \\
\hline Magreb & $272(96,1 \%)$ & $11(3,9 \%)$ & $283(100 \%)$ \\
África subsahariana & $31(93,9 \%)$ & $2(6,1 \%)$ & $33(100 \%)$ \\
Indopakistán & $180(98,9 \%)$ & $2(1,1 \%)$ & $182(100 \%)$ \\
Otros países de Asia & $47(94,0 \%)$ & $3(6,0 \%)$ & $50(100 \%)$ \\
América Central & $55(94,8 \%)$ & $3(5,2 \%)$ & $58(100 \%)$ \\
Caribe & $40(90,1 \%)$ & $4(0,9 \%)$ & $44(100 \%)$ \\
América del Sur & $325(97,9 \%)$ & $7(2,1 \%)$ & $332(100 \%)$ \\
Antigua URSS & $30(93,8 \%)$ & $2(6,2 \%)$ & $32(100 \%)$ \\
Europa del Este & $131(99,2 \%)$ & $1(0,8 \%)$ & $132(100 \%)$ \\
\hline
\end{tabular}

Tabla III. Características de la familia del niño inmigrante en nuestro medio, según zona de procedencia $(n=1.142)$. En algunas familias falta más de un miembro

\begin{tabular}{lcccccc}
\hline Procedencia & $\mathbf{n}$ & $\begin{array}{c}\text { Familia } \\
\text { completa }\end{array}$ & $\begin{array}{c}\text { Familia } \\
\text { incompleta }\end{array}$ & $\begin{array}{c}\text { Falta } \\
\text { padre }\end{array}$ & $\begin{array}{c}\text { Falta } \\
\text { madre }\end{array}$ & $\begin{array}{c}\text { Falta } \\
\text { hermano/os }\end{array}$ \\
\hline Magreb & 283 & $220(77,7 \%)$ & $63(22,3 \%)$ & $25(8,8 \%)$ & $8(2,8 \%)$ & $42(14,8 \%)$ \\
África subsahariana & 33 & $23(69,7 \%)$ & $10(30,3 \%)$ & $4(12,1 \%)$ & $5(15,5 \%)$ & $9(27,3 \%)$ \\
Indopakistán & 183 & $162(88,5)$ & $21(11,5)$ & $3(1,6 \%)$ & $6(3,3 \%)$ & $14(7,7 \%)$ \\
Otros países de Asia & 50 & $40(80 \%)$ & $10(20 \%)$ & $1(2,0 \%)$ & $3(6,0 \%)$ & $7(14,0 \%)$ \\
América Central & 57 & $23(40,4 \%)$ & $34(59,6 \%)$ & $26(45,6 \%)$ & $3(5,3 \%)$ & $11(19,3 \%)$ \\
Caribe & 43 & $21(48,8 \%)$ & $22(51,2 \%)$ & $16(37,2 \%)$ & $5(11,6 \%)$ & $6(14,0 \%)$ \\
América del Sur & 329 & $192(58,4 \%)$ & $137(41,6 \%)$ & $102(31,0 \%)$ & $7(2,1 \%)$ & $43(13,0 \%)$ \\
Antigua URSS & 32 & $17(53,1 \%)$ & $15(46,9 \%)$ & $15(46,9 \%)$ & $0(0 \%)$ & $0(0 \%)$ \\
Europa del Este & 132 & $112(84,8 \%)$ & $20(15,2 \%)$ & $14(10,6 \%)$ & $3(2,3 \%)$ & $5(3,8 \%)$ \\
\hline
\end{tabular}

En un $13,2 \%$ de los casos había consanguinidad entre los padres, de segundo grado (entre primos o entre tíos y sobrinos) o de tercer grado (entre primos segundos o entre tíos segundos y sobrinos segundos). No había casos de consanguinidad de primer grado (entre padres e hijos o entre hermanos). Por zona de procedencia el mayor porcentaje de consanguinidad se encontró entre los inmigrantes de la zona indopa- quistaní (38,6\%), seguidos por los de África subsahariana $(36,7 \%)$ y los marroquíes (20,5\%) (tabla IV). Tanto en África subsahariana como en la zona indopaquistaní fueron más frecuentes las uniones consanguíneas de $2 .^{\circ}$ grado (80\% y 64,2\% respectivamente). En el Magreb un 47,3\% de las consanguinidades fueron de $2 .^{\circ}$ grado, mientras que la mayoría $(52,7 \%)$ lo fueron de 3 . $^{\text {er }}$ grado. 
Tabla IV. Consanguinidad de los padres por zonas de procedencia $(n=1.127)$

\begin{tabular}{lccc}
\hline Procedencia & $\mathbf{n}$ & Padres consanguíneos & Padres no consanguíneos \\
\hline Magreb & 272 & $56(20,5 \%)$ & $216(79,5 \%)$ \\
África subsahariana & 30 & $11(36,7 \%)$ & $19(63,3 \%)$ \\
Indopakistán & 182 & $70(38,6 \%)$ & $112(61,4 \%)$ \\
Otros países de Asia & 49 & $1(1,8 \%)$ & $48(98,2 \%)$ \\
América Central & 55 & $2(3,6 \%)$ & $53(96,4 \%)$ \\
Caribe & 324 & $5(1,8 \%)$ & $319(98,2 \%)$ \\
América del Sur & 43 & $0(0 \%)$ & $43(100 \%)$ \\
Antigua URSS & 32 & $2(6,3 \%)$ & $30(93,7 \%)$ \\
Europa del Este & 128 & $0(0 \%)$ & $128(100 \%)$ \\
\hline
\end{tabular}

Se obtuvieron 987 respuestas sobre la religión de la familia: 453 (45,9\%) se declararon musulmanes, 383 (38,8\%) católicos y $104(10,5 \%)$ de otras confesiones cristianas, perteneciendo el resto: 47 $(4,8 \%)$ a opciones minoritarias. Eran practicantes el $92,1 \%$ de los musulmanes, el 58,7\% de otras confesiones cristianas y el 50,7\% de los católicos. La religión islámica predominaba en Magreb (98,9\%), África subsahariana (81,8\%), Indopakistán $(92,0 \%)$; la católica en América Central (70,4\%), Caribe (90\%), América del Sur $(81,7 \%)$ y otros países de Asia (70,4\%), especialmente en este caso a expensas de los filipinos; en los países de la antigua URSS y en los de Europa del Este las religiones más declaradas se reparten entre católicos (40\% y $17,9 \%$ respectivamente) y otras confesiones cristianas (36\% y 55,4\%).

El nivel de alfabetización y los estudios de los padres por zonas de origen se muestran en las tablas V y VI. En la alfabetización hay una diferencia significativa $(p=0,033)$ entre las mujeres del Magreb (más analfabetismo) y las de Africa subsahariana. En cuanto al nivel de estudios existe una diferencia significativa ( $p$ $<0,001)$ para los dos sexos en relación al lugar de procedencia.

Se consideró el índice de hacinamiento $(\mathrm{IH})$ para conocer alguna característica de las condiciones de vida en nuestro país. Se pudo calcular en 1.180 niños: en el $65,1 \%$ el $\mathrm{IH}$ era menor de 2 y en el 34,9\% mayor de 2 . El mayor hacinamiento se da en familias indopakistaníes $(49,7 \%)$, magrebíes $(41,6 \%)$ y otros países de Asia (41,8\%). De África subsahariana solo un 5,1\% vivían hacinados y en los de la antigua URSS el $9,5 \%$, pero se trata de las procedencias con menor número de niños en el estudio. Cifras intermedias son las de América Central (33,3\%), América del Sur 
Tabla V. Grado de alfabetización de los padres por zonas de procedencia

\begin{tabular}{|c|c|c|c|c|c|c|}
\hline \multirow[t]{2}{*}{ Procedencia } & \multicolumn{3}{|c|}{$\begin{array}{l}\text { ¿Sabe el padre leer } \\
\text { y escribir en su idioma? } \\
\quad(n=1.067)\end{array}$} & \multicolumn{3}{|c|}{$\begin{array}{l}\text { ¿Sabe la madre leer } \\
\text { y escribir en su idioma? } \\
\quad(n=1.100)\end{array}$} \\
\hline & Sí & No & Total & Sí & No & Total \\
\hline Magreb & $210(79,8 \%)$ & $53(20,2 \%)$ & $263(100 \%)$ & $118(44,4 \%)$ & $148(55,6 \%)$ & $266(100 \%)$ \\
\hline África subsahariana & $27(87,1 \%)$ & $4(12,9 \%)$ & $31(100 \%)$ & $20(64,5 \%)$ & $11(35,5 \%)$ & $31(100 \%)$ \\
\hline Indopakistán & $157(89,7 \%)$ & $18(10,3 \%)$ & $175(100 \%)$ & $143(82,7 \%)$ & $30(17,3 \%)$ & $173(100 \%)$ \\
\hline Otros países de Asia & $46(100 \%)$ & $0(0 \%)$ & $46(100 \%)$ & $47(100 \%)$ & $0(0 \%)$ & $47(100 \%)$ \\
\hline América Central & $53(100 \%)$ & $0(0 \%)$ & $53(100 \%)$ & $54(100 \%)$ & $0(0 \%)$ & $54(100 \%)$ \\
\hline Caribe & $38(100 \%)$ & $0(0 \%)$ & $38(100 \%)$ & $42(97,7 \%)$ & $1(2,3 \%)$ & $43(100 \%)$ \\
\hline América del Sur & $309(99,7 \%)$ & $1(0,3 \%)$ & $310(100 \%)$ & $329(99,7 \%)$ & $1(0,3 \%)$ & $330(100 \%)$ \\
\hline Antigua URSS & $29(100 \%)$ & $0(0 \%)$ & $29(100 \%)$ & $29(100 \%)$ & $0(0 \%)$ & $29(100 \%)$ \\
\hline Europa del Este & $122(100 \%)$ & $0(0 \%)$ & $122(100 \%)$ & $124(97,6 \%)$ & $3(2,4 \%)$ & $127(100 \%)$ \\
\hline
\end{tabular}

\begin{tabular}{|c|c|c|c|c|c|c|c|c|}
\hline \multirow[t]{2}{*}{ Procedencia } & \multicolumn{2}{|c|}{ No escolarizado } & \multicolumn{2}{|c|}{$\begin{array}{l}\text { Educación primaria, } \\
\text { secundaria, formación } \\
\text { profesional }\end{array}$} & \multicolumn{2}{|c|}{$\begin{array}{c}\text { Estudios } \\
\text { superiores }\end{array}$} & \multicolumn{2}{|c|}{ Desconocidos } \\
\hline & Padre & Madre & Padre & Madre & Padre & Madre & Padre & Madre \\
\hline Magreb & $5(13,9 \%)$ & $129(50,8 \%)$ & $24(66,7 \%)$ & $102(40,2 \%)$ & $4(11,1 \%)$ & $7(2,8 \%)$ & $3(8,3 \%)$ & $16(3,6 \%)$ \\
\hline África subsahariana & $1(12,5 \%)$ & $10(30,3 \%)$ & $5(62,5 \%)$ & $15(45,5 \%)$ & $1(12,5 \%)$ & $3(9,1 \%)$ & $1(12,5 \%)$ & $5(15,1 \%)$ \\
\hline Indopakistán & $1(4,1 \%)$ & $24(14,2 \%)$ & $20(80 \%)$ & $133(78,7 \%)$ & $3(12 \%)$ & $2(1,2 \%)$ & $1(4 \%)$ & $10(5,9 \%)$ \\
\hline Otros países de Asia & $0(0 \%)$ & $0(0 \%)$ & $4(36,4 \%)$ & $40(66,7 \%)$ & $1(9,1 \%)$ & $8(13,3 \%)$ & $6(54,5 \%)$ & $12(20,0 \%)$ \\
\hline América Central & $0(0 \%)$ & $0(0 \%)$ & $4(57,1 \%)$ & $43(84,3 \%)$ & $3(42,9 \%)$ & $7(13,7 \%)$ & $0(0 \%)$ & $1(2 \%)$ \\
\hline Caribe & $0(0 \%)$ & $2(0,6 \%)$ & $33(80,5 \%)$ & $260(80,7 \%)$ & $7(17,1 \%)$ & $48(14,9 \%)$ & $1(2,4 \%)$ & $12(3,7 \%)$ \\
\hline América del Sur & $0(0 \%)$ & $5(11,6 \%)$ & $1(100 \%)$ & $32(74,4 \%)$ & $0(0 \%)$ & $5(11,6 \%)$ & $0(0 \%)$ & $1(2,3 \%)$ \\
\hline Antigua URSS & $0(0 \%)$ & $0(0 \%)$ & $3(42,9 \%)$ & $17(51,5 \%)$ & $1(14,3 \%)$ & $13(39,4 \%)$ & $3(42,9 \%)$ & $3(9,1 \%)$ \\
\hline Europa del Este & $0(0 \%)$ & $3(2,4 \%)$ & $23(88,5 \%)$ & $112(88,9 \%)$ & $3(11,5 \%)$ & $8(6,3 \%)$ & $0(0 \%)$ & $3(2,4 \%)$ \\
\hline
\end{tabular}

(33\%), Europa del Este $(24,3 \%)$ y Caribe $(18,6 \%)$.

Se estudió el hacinamiento según la población del municipio de residencia; en los municipios de más de 50.000 habitantes el IH fue superior a 2 en el $40,5 \%$ de los casos y en los menores de 50.000 fue superior a 2 en el $28,1 \%$ ( $p$ $<0,001)$.

\section{Discusión}

Nuestra muestra es equivalente en la distribución por sexos a la población catalana menor de 15 años, aunque haya un porcentaje superior de niños que de niñas. Entre la población catalana el $51,47 \%$ son niños y el $48,53 \%$ son niñas. Sí que hay una diferencia significativa (más varones) entre los proceden- 
tes de países asiáticos, lo que sugiere un estilo migratorio diferente, ya que, por ejemplo, en Pakistán, la proporción entre hombres y mujeres es de $52 / 48 \%{ }^{11}$.

Según el Informe sobre la situación social de inmigrantes y refugiados de $2007^{12}$, la percepción de los inmigrantes para acceder a los servicios de salud depende de su país o región de origen, está relacionado con la cultura, las creencias sobre la salud y la enfermedad, y con el sistema sanitario que han vivido. La mujer se preocupa más por la salud y la enfermedad que el hombre y la presencia de hijos lleva a una mayor apertura al sistema de salud. Otras publicaciones ${ }^{13,14}$ exponen que la utilización de los servicios sanitarios está más relacionada con el nivel socioeconómico que con la migración en sí, ya que el comportamiento es el mismo entre inmigrantes y autóctonos del mismo nivel socioeconómico.

En nuestro estudio los niños no tardaron mucho en ir a las consultas pediátricas desde que llegaban de su país. En Cataluña la población inmigrante utiliza fácilmente la red sanitaria pública ${ }^{8}$, a diferencia de otros países como EE. UU. 15,16 , probablemente porque es gratuita, universal para los niños, de fácil acceso y dotada de los recursos necesarios. Además pueden influir otros factores: la demanda por parte de la escuela de un certificado de salud y vacunaciones y el hecho de que la reagrupación familiar suele acontecer cuando se da una cierta estabilidad de los adultos que han llegado primero y que pueden conocer el funcionamiento de nuestra sanidad. En el caso de los niños adoptados, que acudieron antes a las consultas, se suma la recomendación de las Agencias de Adopción y que los padres son autóctonos.

La familia, fundamental para el desarrollo del niño, no está completa entre una tercera y una cuarta parte de los niños estudiados, aunque casi en la totalidad de ellos el cuidador principal es alguno de los progenitores. Destaca el alto porcentaje de niños de América Central y del Sur que tienen a su padre ausente. En los casos en que en la familia nuclear falta alguno de sus miembros se ha de considerar el posible riesgo que ello comporta para la salud psicoafectiva del menor, riesgo que se añade al inevitable duelo migratorio.

Las zonas con mayor índice de consanguinidad son África subsahariana, Indopakistán y Magreb. Con respecto a las familias magrebíes, la cifra de consanguinidad es ligeramente inferior a la encontrada por Cabral y cols. en su estudio ${ }^{17}$. Es importante el conocimiento de este dato de cara al diagnóstico y prevención de enfermedades hereditarias. 
Es de destacar que los que se declaran musulmanes, mayoritariamente los procedentes de Magreb, África subsahariana, Pakistán y Bangladesh, tienen un altísimo porcentaje de práctica religiosa. Teniendo en cuenta que la religión tiene una gran influencia en sus vidas es importante el conocimiento de aquellas prácticas religiosas que pueden condicionar el cuidado de la salud.

Tanto en hombres como en mujeres predominan los estudios primarios, secundarios y formación profesional, excepto en las mujeres magrebíes, de las cuales la mitad no ha ido a la escuela. Si lo comparamos con cifras de España ${ }^{18}$ sobre el nivel de formación de los extranjeros extracomunitarios, que solo diferencia por sexo, en nuestro estudio baja el porcentaje de estudios de cualquier tipo a expensas del aumento del analfabetismo; en el caso de los hombres la diferencia es pequeña pero aumenta bastante en las mujeres.

La alfabetización y el nivel de estudios influyen en la comunicación en la consulta y se han de tener en cuenta al utilizar información escrita o con imágenes. En África es donde hemos encontrado un mayor índice de analfabetismo, con una clara diferencia entre hombres y mujeres, sobre todo en Magreb. Los datos de África subsahariana se han de evaluar con cautela, porque la muestra de esta procedencia es pequeña. En la zona indopakistaní hay también un porcentaje elevado de analfabetismo, superior entre las mujeres pero menor que entre las africanas. En el resto de países la alfabetización es equivalente al nuestro y sin diferencias por sexos. Si comparamos los resultados de nuestro estudio con las cifras publicadas por la Unidad de Estadística de las Naciones Unidas ${ }^{19}$ sobre alfabetización en los países de origen de estos inmigrantes, nuestros resultados son mejores en todos los casos, por lo que podría decirse que las personas que emigran a nuestro país no son los que se encuentran en peor situación.

Un elevado porcentaje de familias de nuestro estudio viven en condiciones de hacinamiento; el hecho de que en los municipios de menos de 50.000 habitantes el hacinamiento sea menor posiblemente sea debido a que en estas poblaciones el precio de la vivienda es más asequible, pudiendo deducirse que el hacinamiento dependería más del lugar donde se establecen que de la procedencia de los inmigrantes.

Limitaciones del estudio: el número de inmigrantes de algunas procedencias y el de respuestas obtenidas en algunas preguntas limita la interpretación de algunos datos. Asimismo, han podido haber erro- 
res en la interpretación de preguntas y respuestas por las dificultades de comunicación, ya que no siempre se ha dispuesto de mediadores o traductores. A pesar de ello creemos, dado que en las instrucciones se aconsejaba dejar en blanco los casos dudosos, que los resultados tienen una validez importante.

\section{Conclusiones}

- La población infantil inmigrante no tarda en llegar a las consultas de AP.

- Algo más de la cuarta parte no tienen la familia nuclear completa, lo que influye en el bienestar psicológico del niño.

- Es importante preguntar por el gra- do de consanguinidad de los padres, especialmente a los procedentes de países donde es más frecuente.

- Se ha de valorar el hacinamiento en el hogar, por lo que puede representar de riesgo para la salud.

- Los niveles de alfabetización y de estudios, especialmente de las mujeres, unidos en algunos casos al idioma, dificultan la comunicación y se han de tener en cuenta al utilizar información escrita.

- Son necesarios más medios y formación para atender a estos niños/as, comunicarse mejor con las familias y hacer frente a los posibles problemas relacionados con su situación familiar.

\section{Bibliografía}

1. López Vélez R, Huerga H. Inmigración y salud; aproximación desde la Atención Primaria. Madrid: Editorial PBM S.L.; 2002.

2. Vall O, Puig JC, García O. La inmigración y su repercusión sanitaria. Aspectos culturales y nuevas patologías emergentes. Pediatr Integral. 2001;6: 42-50.

3. Kinsman SB, Sally M, Fox K. El enfoque multicultural en la práctica pediátrica. Pediatr Rev. 1997;18:30-5.

4. Parella S, Lurbe K. Sociologia de la Immigració. Document de treball Núm. 1. Coordinació Carlota Solé [consultado el 18/07/2009]. Disponible en www.iecat.net/institucio/societats/acsocio logia/publicacions/doc_treball/treb1_immigracio. $\mathrm{htm}$

5. Programa de las Naciones Unidas para el desarrollo. Informe sobre desarrollo humano 2006 [consultado el 18/07/2009]. Disponible en www. undp.org/spanish

6. Ministerio de Trabajo e Inmigración. Anuario Estadístico de Inmigración 2007. Observatorio permanente de la inmigración [consultado el 18/07/ 2009]. Disponible en http://extranjeros.mtas.es/ es/InformacionEstadistica/Anuarios/

7. IDESCAT. Anuari Estadístic de Catalunya [consultado el 18/07/2009]. Disponible en www. idescat.net/cat/idescat/publicacions/

8. Berra S, Elorza Ricart JM, Bartomeu N, Hausmann S, Serra-Sutton $V$, Rajmil L. Necesidades en 
salud y utilización de los servicios sanitarios en la población inmigrante en Cataluña. Revisión exhaustiva de la literatura científica. Barcelona. Agència d'Avaluació de Tecnología i Recerca Mèdiques. CatSalut. Departament de Sanitat i Seguretat Social; 2004. Informe Tècnic N.:IN01/ 2004.

9. Generalitat de Catalunya. Departament de Salut. Protocol d'Atenció a Infants Immigrats. Programa de seguiment del nen sa. Barcelona: Generalitat de Catalunya; 2005 [consultado el 18/07/ 2009]. Disponible en www.gencat.cat/salut/dep salut/pdf/protoimmi2006.pdf

10. Organización Internacional del Trabajo. Hacinamiento del hogar [consultado el 18/07/2009]. Disponible en www.oitcinterfor.org/public/spa nish/region/ampro/cinterfor/temas/youth/doc/jo v_mer/par/iii/index.htm

11. Government of Pakistan [consultado el 18/ 07/2009]. Disponible en www.pakistan.gov.pk/

12. Ministerio de Trabajo e Inmigración. Secretaría de Estado de Inmigración y Emigración. Informe sobre la situación de la integración social de los inmigrantes y refugiados en 2007. Foro para la integración social de los inmigrantes. Madrid: Ministerio de Trabajo e Inmigración; 2007 [consultado el 18/07/2009]. Disponible en www.mtin.es/es/mi graciones/Integracion/Foro/docs/Informe-situa cion-integracion-inmigrantes2007.pdf

13. Cots Reguant F. Servei d'Estudis de I'IMAS. Unitat de Recerca en Serveis Sanitaris IMIM. El "inmigrante económico": ¿se caracteriza más por inmigrante o por económico? Gestión Clin Sanit.
2002;4:134 [consultado el 18/07/2009]. Disponible en http://212.169.42.7/newgenClibPlus/ASP/ printDocument.asp?doclD=GCS14-20\&xs|=Print _ES_PubDoc.xsl www.iiss.es/gcs/gestion14.pdf

14. Rodríguez-García D, San Román Espinosa T. Inmigración, salud y gestión de la diversidad: presentación y avances preliminares de un proyecto en barrios de Cataluña. AIBR. Rev Antropología Iberoamericana. 2007;2:489-520.

15. Yu SM, Huang ZJ, Singh GK. Health status and health services utilization among US Chinese, Asian Indian, Filipino, and other Asian/Pacific Isalnder children. Pediatrics. 2004;113:101-7.

16. Huang ZJ, Yu SM, Ledsky R. Health status and health services access and use among children in U.S. Immigrant families. Am J Public Health. 2006;96:634-40.

17. Cabral Salvadores $M$, Guasch Jordán E, Rifá Pujol MA, Coromina Pou E, Bellvehí Sáenz M, Rufas Cebollero AM. El reto de la inmigración: estudio en Atención Primaria. Rev Pediatr Aten Primaria. 2007;9:17-30.

18. Pajares M. Inmigración y mercado de trabajo. Análisis de datos de España y Cataluña, Informe 2007. Observatorio permanente de la inmigración. Madrid: Ministerio de Trabajo y Asuntos Sociales [consultado el 18/07/2009]. Disponible en www. tt.mtas.es/periodico/inmigracion/200706/infor me.pdf

19. United Nations Statics Division. Indicators on literacy [consultado el 18/07/2009]. Disponible en http://unstats.un.org/unsd/demographic/pro ducts/socind/literacy.htm 